\title{
SCHATTEN NORMS OF TOEPLITZ MATRICES WITH FISHER-HARTWIG SINGULARITIES*
}

\author{
ALBRECHT BÖTTCHER ${ }^{\dagger}$
}

\begin{abstract}
The asymptotics of the Schatten norms of finite Toeplitz matrices generated by functions with a Fisher-Hartwig singularity are described as the matrix dimension $n$ goes to infinity. The message of the paper is to reveal some kind of a kink: the $p$ th Schatten norm increases as $n$ to the power $1 / p$ before the singularity reaches a critical point and as $n$ to an exponent depending on the singularity beyond the critical point.
\end{abstract}

Key words. Toeplitz matrix, Schatten norm, Fisher-Hartwig singularity, Avram-Parter theorem, Szegö theorem.

AMS subject classifications. 47B35, 15A60.

1. Introduction. Let $a$ be a function in $L^{1}(-\pi, \pi)$ and let $\left\{a_{n}\right\}_{n \in \mathbb{Z}}$ be the sequence of the Fourier coefficients of $a$,

$$
a_{n}=\int_{-\pi}^{\pi} a(x) e^{-i n x} \frac{d x}{2 \pi} .
$$

We denote by $T_{n}(a)$ the $n \times n$ Toeplitz matrix $\left(a_{j-k}\right)_{j, k=1}^{n}$. For $1 \leq p \leq \infty$, the Schatten norm $\left\|T_{n}(a)\right\|_{p}$ is defined by

$$
\left\|T_{n}(a)\right\|_{p}:= \begin{cases}\left(\sum_{j=1}^{n} s_{j}^{p}\left(T_{n}(a)\right)\right)^{1 / p} & \text { for } \quad 1 \leq p<\infty, \\ s_{n}\left(T_{n}(a)\right) & \text { for } \quad p=\infty,\end{cases}
$$

where $s_{1}\left(T_{n}(a)\right) \leq \ldots \leq s_{n}\left(T_{n}(a)\right)$ are the singular values of $T_{n}(a)$. This paper addresses the behavior of the Schatten norms $\left\|T_{n}(a)\right\|_{p}$ as $n \rightarrow \infty$ in the case where $a$ is a function with a singularity of the Fisher-Hartwig type. An archetypal example of such a function is

$$
\omega_{\alpha}^{+}(x)= \begin{cases}0 & \text { for } \quad x \in(-\pi, 0) \\ 1 / x^{\alpha} & \text { for } \quad x \in(0, \pi)\end{cases}
$$

where $0<\alpha<1$. One can show that there exist constants $C_{1}(\alpha), C_{\infty}(\alpha) \in(0, \infty)$ depending only on $\alpha$ such that

$$
\left\|T_{n}\left(\omega_{\alpha}^{+}\right)\right\|_{1} \sim C_{1}(\alpha) n, \quad\left\|T_{n}\left(\omega_{\alpha}^{+}\right)\right\|_{\infty} \sim C_{\infty}(\alpha) n^{\alpha} .
$$

Here and in what follows $x_{n} \sim y_{n}$ means that $x_{n} / y_{n} \rightarrow 1$ as $n \rightarrow \infty$. Thus, the exponent of $n$ in the asymptotics of the trace norm $\left\|T_{n}\left(\omega_{\alpha}^{+}\right)\right\|_{1}$ is independent of $\alpha$, while

\footnotetext{
*Received by the editors 29 June 2006. Accepted for publication 13 September 2006. Handling Editor: Shmuel Friedland.

${ }^{\dagger}$ Fakultät für Mathematik, Technische Universität Chemnitz, D - 09107 Chemnitz, Germany (aboettch@mathematik.tu-chemnitz.de).
} 
this exponent depends heavily on $\alpha$ for the spectral norm $\left\|T_{n}\left(\omega_{\alpha}^{+}\right)\right\|_{\infty}$. Computing Frobenius norms we get

$$
\left\|T_{n}\left(\omega_{\alpha}^{+}\right)\right\|_{2} \sim \begin{cases}C_{2}(\alpha) n^{1 / 2} & \text { for } \quad \alpha<1 / 2, \\ C_{2}(\alpha)(n \log n)^{1 / 2} & \text { for } \quad \alpha=1 / 2, \\ C_{2}(\alpha) n^{\alpha} & \text { for } \quad \alpha>1 / 2\end{cases}
$$

with constants $C_{2}(\alpha) \in(0, \infty)$. This time we observe a kind of a kink at $\alpha=1 / 2$ : for $\alpha<1 / 2$ the exponent of $n$ is independent of $\alpha$ and for $\alpha>1 / 2$ the asymptotics of $\left\|T_{n}\left(\omega_{\alpha}^{+}\right)\right\|_{2}$ is governed by $\alpha$.

Theorems of the Szegö-Avram-Parter type state that

$$
\lim _{n \rightarrow \infty} \frac{1}{n} \sum_{j=1}^{n} F\left(s_{j}\left(T_{n}(a)\right)\right)=\int_{-\pi}^{\pi} F(|a(x)|) \frac{d x}{2 \pi}
$$

for appropriate functions $F:[0, \infty) \rightarrow \mathbb{R}$. The functions $F$ are usually referred to as test functions. The Avram-Parter theorem says that (1.1) is true for every $F \in C[0, \infty)$ if $a$ belongs to $L^{\infty}(-\pi, \pi)$ (see [1], [4]; a full proof is also in [2]). Under the sole assumption that $a$ be in $L^{1}(-\pi, \pi)$, Tyrtyshnikov and Zamarashkin [8], [9] proved (1.1) for all bounded and uniformly continuous functions $F$. A textbook proof of the Tyrtyshnikov-Zamarashkin theorem is in Tilli's paper [7]. The quotient $\left\|T_{n}(a)\right\|_{p}^{p} / n$ is just the left-hand side of (1.1) for $F(s)=s^{p}$. This function is neither bounded nor uniformly continuous, but Serra Capizzano [5] showed that nevertheless (1.1) is valid in this case, that is, after abbreviating $L^{p}(-\pi, \pi)$ to $L^{p}$ and letting

$$
\|a\|_{p}:=\left(\int_{-\pi}^{\pi}|a(x)|^{p} \frac{d x}{2 \pi}\right)^{1 / p}
$$

we have

$$
\lim _{n \rightarrow \infty} \frac{\left\|T_{n}(a)\right\|_{p}}{n^{1 / p}}=\left\{\begin{array}{lll}
\|a\|_{p} & \text { if } & a \in L^{p} \\
\infty & \text { if } & a \notin L^{p}
\end{array}\right.
$$

Since $\omega_{\alpha}^{+}$is in $L^{p}$ if and only if $p<1 / \alpha$, we deduce that

$$
\lim _{n \rightarrow \infty} \frac{\left\|T_{n}\left(\omega_{\alpha}^{+}\right)\right\|_{p}}{n^{1 / p}}=\left\{\begin{array}{lll}
\left\|\omega_{\alpha}^{+}\right\|_{p} & \text { if } & p<1 / \alpha \\
\infty & \text { if } & p \geq 1 / \alpha .
\end{array}\right.
$$

This is the explanation of the kink.

Formula (1.2) does not describe the order of the growth of $\left\|T_{n}(a)\right\|_{p}$ for $a \in L^{1} \backslash L^{p}$. We here tackle this question for a special but sufficiently interesting class of functions $a \in L^{1}$. For $0<\alpha<1$, define $\omega_{\alpha}^{+}(x)$ as above and put $\omega_{\alpha}^{-}(x)=\omega_{\alpha}^{+}(-x)$. We consider functions of the form

$$
a(x)=\omega_{\beta}^{-}(x) b(x)+\omega_{\gamma}^{+}(x) c(x)
$$


where $0<\beta<1,0<\gamma<1, b \in L^{\infty}, c \in L^{\infty}$. For example, our analysis includes the function

$$
a(x)=\frac{1}{|x|^{\alpha}}=\omega_{\alpha}^{-}(x)+\omega_{\alpha}^{+}(x),
$$

the function

$$
a(x)=\left|e^{i x}-1\right|^{-\alpha}=\left(2\left|\sin \frac{x}{2}\right|\right)^{-\alpha}=\left[\omega_{\alpha}^{-}(x)+\omega_{\alpha}^{+}(x)\right] \frac{|x|^{\alpha}}{(2|\sin (x / 2)|)^{\alpha}},
$$

and also such functions as

$$
a(x)= \begin{cases}\left|e^{i x}-1\right|^{-\beta} \exp (i \delta(x-\pi)) & \text { for } \quad x<0 \\ \left|e^{i x}-1\right|^{-\gamma} \exp (i \eta(\pi-x)) & \text { for } \quad x>0\end{cases}
$$

where $\alpha, \beta, \gamma \in(0,1)$ and $\delta, \eta \in \mathbb{C}$. The class (1.3) includes in particular all functions with a single Fisher-Hartwig singularity, that is, all functions of the form (1.4) with $\beta=\gamma$ (see, e.g., [2]). The following result provides us with upper bounds.

TheOrem 1.1. Let $a$ be of the form (1.3) with $0<\beta<1,0<\gamma<1, b \in L^{\infty}$, $c \in L^{\infty}$ and put $\alpha=\max (\beta, \gamma)$. If $1 / \alpha \leq p \leq \infty$, then there exists a constant $C_{p}(a) \in(0, \infty)$ such that

$$
\left\|T_{n}(a)\right\|_{p} \leq \begin{cases}C_{p}(a) n^{\alpha}(\log n)^{1+\alpha} & \text { if } p=1 / \alpha, \\ C_{p}(a) n^{\alpha} \log n & \text { if } 1 / \alpha<p<\infty \\ C_{p}(a) n^{\alpha} & \text { if } p=\infty\end{cases}
$$

for all $n \geq 1$.

To get lower bounds, we need some technical assumptions. Here is our result.

TheOREm 1.2. Let $a$ be of the form (1.3) with $0<\beta<1,0<\gamma<1, b \in L^{\infty}$, $c \in L^{\infty}$. Suppose $b$ and $c$ are one-sided Lipschitz continuous at 0 , that is, the one-sided limits $b(0-0)$ and $c(0+0)$ exist and

$$
\begin{aligned}
& |b(x)-b(0-0)|=O(|x|) \quad \text { as } \quad x \rightarrow 0-0 \\
& |c(x)-c(0+0)|=O(|x|) \quad \text { as } \quad x \rightarrow 0+0 .
\end{aligned}
$$

Put $\alpha=\max (\beta, \gamma)$ and assume

$$
\begin{aligned}
& b(0-0) \neq 0 \quad \text { if } \quad \alpha=\beta>\gamma, \\
& c(0+0) \neq 0 \quad \text { if } \quad \alpha=\gamma>\beta, \\
& |b(0-0)|+|c(0+0)|>0 \quad \text { if } \quad \alpha=\beta=\gamma .
\end{aligned}
$$

If $1 / \alpha<p \leq \infty$, then there exists a constant $K(a) \in(0, \infty)$ depending only on a such that

$$
K(a) n^{\alpha} \leq\left\|T_{n}(a)\right\|_{p} \text { for all } n \geq 1 .
$$


If $p=1 / \alpha$ and $b$ and $c$ are in $C^{1}[-\pi, \pi]$, then there is a constant $K(a) \in(0, \infty)$ depending only on a such that

$$
K(a) n^{\alpha} \leq\left\|T_{n}(a)\right\|_{p} \text { for all } n \geq 1 .
$$

We will prove these two theorems in Sections 2 and 3. The idea of the proof is very simple. The lower bounds follow from the inequality $\left\|T_{n}(a)\right\|_{p} \geq\left\|T_{n}(a)\right\|_{\infty}$ and the result of [3] for the norm $\|\cdot\|_{\infty}$. To obtain the upper bounds we take into account that $T_{n}(a)=T_{n}\left(s_{n} a\right)$, where $s_{n} a$ is the $(n-1)$ st partial sum of the Fourier series of $a$, we use the inequality

$$
\left\|T_{n}(a)\right\|_{p} \leq n^{1 / p}\|a\|_{p},
$$

which was shown by Serra Capizzano and Tilli [6] to be true for all $a \in L^{p}, 1 \leq p<\infty$, $n \geq 1$ to get $\left\|T_{n}(a)\right\|_{p} \leq n^{1 / p}\left\|s_{n} a\right\|_{p}$, and we finally employ the representation of $s_{n} a$ via the Dirichlet kernel to estimate $\left\|s_{n} a\right\|_{p}$.

We conjecture that for $1 / \alpha \leq p<\infty$ the stronger estimates

$$
\begin{aligned}
& K_{p}(a) n^{\alpha}(\log n)^{\alpha} \leq\left\|T_{n}(a)\right\|_{p} \leq C_{p}(a) n^{\alpha}(\log n)^{\alpha} \quad \text { if } \quad p=1 / \alpha, \\
& K_{p}(a) n^{\alpha} \leq\left\|T_{n}(a)\right\|_{p} \leq C_{p}(a) n^{\alpha} \quad \text { if } \quad p>1 / \alpha
\end{aligned}
$$

hold and that one can remove the hypothesis that $b$ and $c$ be in $C^{1}[-\pi, \pi]$ in the case $p=1 / \alpha$, but this is still unresolved.

Clearly, combining the inequality

$$
\left\|T_{n}(f)\right\|_{p}-\left\|T_{n}(g)\right\|_{p} \leq\left\|T_{n}(f+g)\right\|_{p} \leq\left\|T_{n}(f)\right\|_{p}+\left\|T_{n}(g)\right\|_{p}
$$

with Theorems 1.1 and 1.2 we obtain estimates for $\left\|T_{n}(a)\right\|_{p}$ if $a$ is of the more general form

$$
a(x)=\sum_{r=1}^{R}\left[\omega_{\beta_{r}}\left(x-x_{r}\right) b_{r}\left(x-x_{r}\right)+\omega_{\gamma_{r}}\left(x-x_{r}\right) c_{r}\left(x-x_{r}\right)\right]
$$

where $\beta_{r}, \gamma_{r}, b_{r}, c_{r}$ are as above.

2. The pure singularity. Let $a=\omega_{\alpha}^{-} b+\omega_{\alpha}^{+} c$ with $0<\alpha<1$ and with constants $b, c \in \mathbb{C}$. We exclude the uninteresting case $b=c=0$. Put

$$
\begin{aligned}
& U(\alpha)=\int_{0}^{\infty} \frac{\cos y}{y^{\alpha}} \frac{d y}{2 \pi}=\frac{1}{4 \Gamma(\alpha) \cos (\pi \alpha / 2)} \\
& V(\alpha)=\int_{0}^{\infty} \frac{\sin y}{y^{\alpha}} \frac{d y}{2 \pi}=\frac{1}{4 \Gamma(\alpha) \sin (\pi \alpha / 2)} .
\end{aligned}
$$

For $n \geq 1$, the Fourier coefficients of $\omega_{\alpha}^{+}$are

$$
\left(\omega_{\alpha}^{+}\right)_{n}=\int_{0}^{\pi} \frac{e^{-i n x}}{x^{\alpha}} \frac{d x}{2 \pi}=n^{\alpha-1} \int_{0}^{n \pi} \frac{e^{-i y}}{y^{\alpha}} \frac{d y}{2 \pi}=n^{\alpha-1}(U(\alpha)-i V(\alpha)+o(1)),
$$


and analogously,

$$
\left(\omega_{\alpha}^{+}\right)_{-n}=n^{\alpha-1}(U(\alpha)+i V(\alpha)+o(1)), \quad\left(\omega_{\alpha}^{-}\right)_{ \pm n}=\left(\omega_{\alpha}^{+}\right)_{\mp n} .
$$

Thus,

$$
\left(\omega_{\alpha}^{-} b+\omega_{\alpha}^{+} c\right)_{ \pm n}=Q_{ \pm} n^{\alpha-1}(1+o(1)) \quad \text { with } \quad Q_{ \pm}=(b+c) U(\alpha) \pm i(b-c) V(\alpha) .
$$

Let $K_{\alpha, b, c}$ be the integral operator on $L^{2}(0,1)$ given by

$$
\left(K_{\alpha, b, c} f\right)(x)=\int_{0}^{1} k(x, y) f(y) d y, \quad x \in(0,1)
$$

with

$$
k(x, y)=\left\{\begin{array}{lll}
Q_{+}(x-y)^{\alpha-1} & \text { for } \quad x>y \\
Q_{-}(y-x)^{\alpha-1} & \text { for } \quad x<y
\end{array}\right.
$$

This operator is bounded and we denote its norm by $\left\|K_{\alpha, b, c}\right\|$. It is clear that $\left\|K_{\alpha, b, c}\right\|>0$ unless $b=c=0$.

THEOREM 2.1. We have

$$
\left\|T_{n}\left(\omega_{\alpha}^{-} b+\omega_{\alpha}^{+} c\right)\right\|_{\infty} \sim\left\|K_{\alpha, b, c}\right\| n^{\alpha} .
$$

Proof. This follows from Theorem 2.4 of [3].

Corollary 2.2. Let $\beta, \gamma \in(0,1)$ and suppose $\beta \neq \gamma$. Then

$$
\left\|T_{n}\left(\omega_{\beta}^{-} b+\omega_{\gamma}^{+} c\right)\right\|_{\infty} \sim \begin{cases}\left\|K_{\beta, b, 0}\right\| n^{\beta} & \text { if } \beta>\gamma \text { and } b \neq 0, \\ \left\|K_{\gamma, 0, c}\right\| n^{\beta} & \text { if } \gamma>\beta \text { and } c \neq 0 .\end{cases}
$$

Proof. Straightforward from Theorem 2.1.

Theorem 2.3. If $1 \leq p<\infty$ and $0<\alpha<1$, then

$$
\left\|T_{n}\left(\omega_{\alpha}^{+}\right)\right\|_{p}=\left\{\begin{array}{lll}
O\left(n^{1 / p} \log n\right) & \text { for } & p<1 / \alpha \\
O\left(n^{\alpha}(\log n)^{1+\alpha}\right) & \text { for } & p=1 / \alpha \\
O\left(n^{\alpha} \log n\right) & \text { for } & p>1 / \alpha
\end{array}\right.
$$

Proof. Let $s_{n} \omega_{\alpha}^{+}$be the $(n-1)$ st partial sum of the Fourier series of $\omega_{\alpha}^{+}$,

$$
\left(s_{n} \omega_{\alpha}^{+}\right)(x)=\sum_{k=-(n-1)}^{n-1}\left(\omega_{\alpha}^{+}\right)_{k} e^{i k x} .
$$

Obviously, $T_{n}\left(\omega_{\alpha}^{+}\right)=T_{n}\left(s_{n} \omega_{\alpha}^{+}\right)$. From (1.5) we therefore deduce that

$$
\left\|T_{n}\left(\omega_{\alpha}^{+}\right)\right\|_{p} \leq n^{1 / p}\left\|s_{n} \omega_{\alpha}^{+}\right\|_{p} .
$$


Put $N=n-1 / 2$. Then in terms of the Dirichlet kernel,

$$
\left(s_{n} \omega_{\alpha}^{+}\right)(x)=\int_{-\pi}^{\pi} \omega_{\alpha}^{+}(t) \frac{\sin N(x-t)}{\sin ((x-t) / 2)} \frac{d t}{2 \pi}=\int_{0}^{\pi} \frac{1}{t^{\alpha}} \frac{\sin N(x-t)}{\sin ((x-t) / 2)} \frac{d t}{2 \pi} .
$$

Consequently,

$$
\left|\left(s_{n} \omega_{\alpha}^{+}\right)(x)\right| \leq C_{1} \int_{0}^{\pi} \frac{1}{t^{\alpha}} \frac{|\sin N(x-t)|}{|x-t|} d t .
$$

Here and in what follows $C_{j}$ denotes a constant in $(0, \infty)$ that is independent of $N$. Substituting $N x=y$ and $N t=\tau$ we get

$$
\left|\left(s_{n} \omega_{\alpha}^{+}\right)(y / N)\right| \leq C_{1} N^{\alpha} \int_{0}^{N \pi} \frac{|\sin (y-\tau)|}{\tau^{\alpha}|y-\tau|} d \tau .
$$

If $-2 \leq y<0$, the integral in $(2.3)$ is

$$
\begin{aligned}
& \int_{0}^{N \pi} \frac{|\sin (\tau+|y|)|}{\tau+|y|} \frac{d \tau}{\tau^{\alpha}}=\int_{0}^{1} \frac{|\sin (\tau+|y|)|}{\tau+|y|} \frac{d \tau}{\tau^{\alpha}}+\int_{1}^{N \pi} \frac{|\sin (\tau+|y|)|}{\tau+|y|} \frac{d \tau}{\tau^{\alpha}} \\
& \leq \int_{0}^{1} \frac{d \tau}{\tau^{\alpha}}+\int_{1}^{\infty} \frac{d \tau}{\tau^{\alpha+1}}=C_{2},
\end{aligned}
$$

and for $y<-2$ the same integral is

$$
\begin{aligned}
& \int_{0}^{N \pi} \frac{|\sin (\tau+|y|)|}{\tau+|y|} \frac{d \tau}{\tau^{\alpha}}=\int_{0}^{|y|} \frac{|\sin (\tau+|y|)|}{\tau+|y|} \frac{d \tau}{\tau^{\alpha}}+\int_{|y|}^{N \pi} \frac{|\sin (\tau+|y|)|}{\tau+|y|} \frac{d \tau}{\tau^{\alpha}} \\
& \leq \int_{0}^{|y|} \frac{d \tau}{|y| \tau^{\alpha}}+\int_{|y|}^{\infty} \frac{d \tau}{\tau^{\alpha+1}}=\frac{1}{(1-\alpha)|y|^{\alpha}}+\frac{1}{\alpha|y|^{\alpha}}=\frac{C_{3}}{|y|^{\alpha}}
\end{aligned}
$$

So let $y>0$. We split the integral in (2.3) into $\int_{0}^{y}$ and $\int_{y}^{N \pi}$. The substitution $\tau=y \xi$ yields

$$
\int_{0}^{y} \frac{|\sin (y-\tau)|}{\tau^{\alpha}|y-\tau|} d \tau=\frac{1}{y^{\alpha}} \int_{0}^{1} \frac{|\sin y(1-\xi)|}{1-\xi} \frac{d \xi}{\xi^{\alpha}}=\frac{1}{y^{\alpha}} \int_{0}^{1} \frac{|\sin y \xi|}{\xi} \frac{d \xi}{(1-\xi)^{\alpha}}
$$

If $y \leq 2$, then

$$
\int_{0}^{1} \frac{|\sin y \xi|}{\xi} \frac{d \xi}{(1-\xi)^{\alpha}} \leq \int_{0}^{1} 2 \frac{d \xi}{(1-\xi)^{\alpha}}=C_{4}
$$

and if $y>2$, we have

$$
\begin{aligned}
& \int_{1 / 2}^{1} \frac{|\sin y \xi|}{\xi} \frac{d \xi}{(1-\xi)^{\alpha}} \leq \int_{1 / 2}^{1} \frac{d \xi}{\xi(1-\xi)^{\alpha}}=C_{5}, \\
& \int_{0}^{1 / y} \frac{|\sin y \xi|}{\xi} \frac{d \xi}{(1-\xi)^{\alpha}} \leq 2^{\alpha} \int_{0}^{1 / y} \frac{|\sin y \xi|}{\xi} d \xi \leq 2^{\alpha} y \int_{0}^{1 / y} d \xi=2^{\alpha}=C_{6}, \\
& \int_{1 / y}^{1 / 2} \frac{|\sin y \xi|}{\xi} \frac{d \xi}{(1-\xi)^{\alpha}} \leq 2^{\alpha} \int_{1 / y}^{1 / 2} \frac{d \xi}{\xi}=2^{\alpha}(\log y-\log 2) \leq C_{7} \log y .
\end{aligned}
$$


Inserting this into (2.6) we obtain

$$
\int_{0}^{y} \frac{|\sin (y-\tau)|}{\tau^{\alpha}|y-\tau|} d \tau \leq C_{8} \frac{\log y}{y^{\alpha}}
$$

for $y>2$. To estimate the remaining integral we substitute $\tau-y=y \xi$ and get

$$
\begin{aligned}
& \int_{y}^{N \pi} \frac{|\sin (y-\tau)|}{\tau^{\alpha}|y-\tau|} d \tau=\int_{0}^{(N \pi-y) / y} \frac{|\sin y \xi|}{y \xi(1+y)^{\alpha}} \frac{d \xi}{\xi^{\alpha}} \leq \int_{0}^{\infty} \frac{|\sin y \xi|}{y \xi(1+y)^{\alpha}} \frac{d \xi}{\xi^{\alpha}} \\
& \leq \frac{1}{(1+y)^{\alpha}} \int_{0}^{1} \frac{d \xi}{\xi}+\frac{1}{y(1+y)^{\alpha}} \int_{1}^{\infty} \frac{d \xi}{\xi^{\alpha+1}} \leq \frac{C_{9}}{y^{\alpha}}
\end{aligned}
$$

In summary, (2.3) combined with (2.4), (2.7) on the one hand and (2.5), (2.8), (2.9) on the other gives

$$
\left|\left(s_{n} \omega_{\alpha}^{+}\right)(y / N)\right| \leq \begin{cases}C_{10} N^{\alpha} & \text { for } \quad|y| \leq 2, \\ C_{11} N^{\alpha}(\log |y|) /|y|^{\alpha} & \text { for } \quad 2<|y|<N \pi .\end{cases}
$$

It follows that

$$
\begin{aligned}
& \left\|s_{n} \omega_{\alpha}^{+}\right\|_{p}^{p}=\int_{-\pi}^{\pi}\left|\left(s_{n} \omega_{\alpha}^{+}\right)(x)\right|^{p} \frac{d x}{2 \pi}=\frac{1}{N} \int_{-N \pi}^{N \pi}\left|\left(s_{n} \omega_{\alpha}^{+}\right)(y / N)\right|^{p} \frac{d y}{2 \pi} \\
& \leq N^{\alpha p-1}\left(2 C_{10}^{p} \int_{0}^{2} \frac{d y}{2 \pi}+2 C_{11}^{p} \int_{2}^{N \pi} \frac{(\log y)^{p}}{y^{\alpha p}} \frac{d y}{2 \pi}\right) .
\end{aligned}
$$

Since

$$
\int_{2}^{N \pi} \frac{(\log y)^{p}}{y^{\alpha p}} d y=\left\{\begin{array}{lll}
O\left(N^{1-\alpha p}(\log N)^{p}\right) & \text { for } & \alpha p<1, \\
O\left((\log N)^{1+p}\right) & \text { for } & \alpha p=1, \\
O\left((\log N)^{p}\right) & \text { for } & \alpha p>1,
\end{array}\right.
$$

we arrive at the desired estimates for $\left\|s_{n} \omega_{\alpha}^{+}\right\|_{p}$.

Corollary 2.4. If $1 \leq p<\infty$ and $0<\alpha<1$, then

$$
\begin{aligned}
& \left\|T_{n}\left(\omega_{\alpha}^{+}\right)\right\|_{p} \sim\left\|\omega_{\alpha}^{+}\right\|_{p} n^{1 / p} \quad \text { for } \quad p<1 / \alpha, \\
& K(\alpha) n^{\alpha} \leq\left\|T_{n}\left(\omega_{\alpha}^{+}\right)\right\|_{p} \leq C_{p}(\alpha) n^{\alpha}(\log n)^{1+\alpha} \quad \text { for } \quad p=1 / \alpha, \\
& K(\alpha) n^{\alpha} \leq\left\|T_{n}\left(\omega_{\alpha}^{+}\right)\right\|_{p} \leq C_{p}(\alpha, p) n^{\alpha}(\log n) \quad \text { for } \quad p>1 / \alpha .
\end{aligned}
$$

Proof. The result for $p<1 / \alpha$ follows from (1.2). In the case $p \geq 1 / \alpha$, the upper bounds are a consequence of Theorem 2.3, while the lower bounds result from the inequality $\left\|T_{n}\left(\omega_{\alpha}^{+}\right)\right\|_{p} \geq\left\|T_{n}\left(\omega_{\alpha}^{+}\right)\right\|_{\infty}$ and Theorem 2.1.

Note that (1.2) implies that the $O\left(n^{1 / p} \log n\right)$ for $p<1 / \alpha$ in Theorem 2.3 may actually be replaced by $O\left(n^{1 / p}\right)$; we used this in the proof of Corollary 2.4. If $\alpha>1 / 2$ and $p \geq 2$, we have

$$
\left\|T_{n}\left(\omega_{\alpha}^{+}\right)\right\|_{p} \leq\left\|T_{n}\left(\omega_{\alpha}^{+}\right)\right\|_{2}=O\left(n^{\alpha}\right)
$$


which provides a better estimate than Theorem 1.1 or Corollary 2.4. Finally, since $\omega_{\alpha}^{+} \in L^{1 /(\alpha+\varepsilon)}$ for each $\varepsilon>0$, we obtain from (1.2) that if $p>1 / \alpha$, then

$$
\left\|T_{n}\left(\omega_{\alpha}^{+}\right)\right\|_{p} \leq\left\|T_{n}\left(\omega_{\alpha}^{+}\right)\right\|_{1 /(\alpha+\varepsilon)} \leq C_{p}(a, \varepsilon) n^{\alpha+\varepsilon}
$$

for all sufficiently large $n$. This is weaker than Theorem 1.1 and Corollary 2.4 but can be derived without the Dirichlet kernel estimates of the proof of Theorem 2.3.

3. Proof of the main result. We are now in a position to prove Theorems 1.1 and 1.2 .

Serra Capizzano and Tilli [6] proved that if $f \in L^{\infty}$ and $g \in L^{1}$, then

$$
\left\|T_{n}(f g)\right\|_{p} \leq\|f\|_{\infty}\left\|T_{n}(|g|)\right\|_{p} .
$$

Consequently,

$$
\left\|T_{n}\left(\omega_{\beta}^{-} b+\omega_{\gamma}^{+} c\right)\right\|_{p} \leq\|b\|_{\infty}\left\|T_{n}\left(\omega_{\beta}^{-}\right)\right\|_{p}+\|c\|_{\infty}\left\|T_{n}\left(\omega_{\gamma}^{+}\right)\right\|_{p},
$$

and Corollary 2.2 and Theorem 2.3 therefore yield Theorem 1.1.

We turn to the proof of Theorem 1.2. So let $1 / \alpha \leq p \leq \infty$. We have

$$
a=\omega_{\beta}^{-} b(0-0)+\omega_{\gamma}^{+} c(0+0)+\omega_{\beta}^{-}(b-b(0-0))+\omega_{\gamma}^{+}(c-c(0+0)) .
$$

Since

$$
\omega_{\gamma}^{+}(x)(c(x)-c(0+0))=\frac{1}{x^{\gamma}} O(x)=O\left(x^{1-\gamma}\right),
$$

the function $\omega_{\gamma}^{+}(c-c(0+0))$ is in $L^{\infty}$ and hence, by the Avram-Parter theorem (or by (3.1) combined with (1.5)),

$$
\left\|T_{n}\left(\omega_{\gamma}^{+}(c-c(0+0))\right)\right\|_{p}=O\left(n^{1 / p}\right)
$$

Analogously, $\left\|T_{n}\left(\omega_{\beta}^{-}(b-b(0-0))\right)\right\|_{p}=O\left(n^{1 / p}\right)$, which implies

$$
\left\|T_{n}(a)\right\|_{p}=\left\|T_{n}\left(\omega_{\beta}^{-} b(0-0)+\omega_{\gamma}^{+} c(0+0)\right)\right\|_{p}+O\left(n^{1 / p}\right) .
$$

Theorem 2.1 and Corollary 2.2 in conjunction with the inequality $\left\|T_{n}\right\|_{p} \geq\left\|T_{n}\right\|_{\infty}$ now yield the assertion for $1 / \alpha<p \leq \infty$.

We are left with the case $p=1 / \alpha$. If the two functions $\omega_{\beta}^{-}(b-b(0-0))$ and $\omega_{\gamma}^{+}(c-c(0+0))$ are in $C^{1}[-\pi, \pi]$, then their Fourier coefficients are $O(1 / n)$ because

$$
\int_{-\pi}^{\pi} f(x) e^{-i n x} d x=\left.\frac{f(x) e^{-i n x}}{-i n}\right|_{-\pi} ^{\pi}+\frac{1}{i n} \int_{-\pi}^{\pi} f^{\prime}(x) e^{-i n x} d x .
$$

Consequently, from the computation at the beginning of Section 3 we obtain

$$
\begin{aligned}
a_{ \pm n} & =b(0-0)\left(\omega_{\beta}^{-}\right)_{n}+c(0+0)\left(\omega_{\gamma}^{+}\right)_{n}+O(1 / n) \\
& =Q_{ \pm} n^{\alpha-1}(1+o(1))+O(1 / n) \\
& =Q_{ \pm} n^{\alpha-1}(1+o(1))+o\left(n^{\alpha-1}\right)
\end{aligned}
$$


with

$$
Q_{ \pm}= \begin{cases}(b(0-0)+c(0+0)) U(\alpha) \pm i(b(0-0)-c(0+0)) V(\alpha) & \text { for } \beta=\gamma=\alpha \\ b(0-0) U(\alpha) \pm i b(0-0) V(\alpha) & \text { for } \beta=\alpha>\gamma \\ c(0+0) U(\alpha) \mp i c(0+0) V(\alpha) & \text { for } \gamma=\alpha>\beta\end{cases}
$$

In either case $Q_{ \pm} \neq 0$. Thus, if we define $K_{\alpha, b, c}$ by $(2.1),(2.2)$, then $\left\|K_{\alpha, b, c}\right\|>0$. Since $a_{ \pm n}=Q_{ \pm} n^{\alpha-1}(1+o(1))$, Theorem 2.4 of [3] yields $\left\|T_{n}(a)\right\|_{\infty} \sim\left\|K_{\alpha, b, c}\right\| n^{\alpha}$. This gives $\left\|T_{n}(a)\right\|_{p} \geq\left\|T_{n}(a)\right\|_{\infty} \geq K(a) n^{\alpha}$ with some constant $K(a)>0$ and completes the proof of Theorem 1.2.

\section{REFERENCES}

[1] F. Avram. On bilinear forms in Gaussian random variables and Toeplitz matrices. Probab. Theory Related Fields, 79:37-45, 1988.

[2] A. Böttcher and B. Silbermann. Introduction to Large Truncated Toeplitz Matrices. Universitext, Springer, New York, 1999.

[3] A. Böttcher and J. Virtanen. Norms of Toeplitz matrices with Fisher-Hartwig symbols. Preprint, arXiv: math.FA/0606016, 2006.

[4] S. V. Parter. On the distribution of the singular values of Toeplitz matrices. Linear Algebra Appl., 80:115-130, 1986.

[5] S. Serra Capizzano. Test functions, growth conditions and Toeplitz matrices. In: Proceedings of the Fourth International Conference on Functional Analysis and Approximation Theory, Vol. II (Potenza, 2000), Rend. Circ. Mat. Palermo (2) Suppl., 68:791-795, 2002.

[6] S. Serra Capizzano and P. Tilli. On unitarily invariant norms of matrix-valued linear positive operators. J. Inequal. Appl., 7:309-330, 2002.

[7] P. Tilli. A note on the spectral distribution of Toeplitz matrices. Linear and Multilinear Algebra, 45:147-159, 1998.

[8] E. E. Tyrtyshnikov. A unifying approach to some old and new theorems on distribution and clustering. Linear Algebra Appl., 232:1-43, 1996.

[9] N. L. Zamarashkin and E. E. Tyrtyshnikov. Distribution of the eigenvalues and singular numbers of Toeplitz matrices under weakened requirements on the generating function. $S b$. Math., 188:1191-1201, 1997. 